\title{
Solitary Schwannoma on the Hypothenar Region of the Hand: A Literature Review
}

\author{
Kastanis G ${ }^{* 1}$, Kapsetakis $P^{1}$, Magarakis G ${ }^{1}$, Bachlitzanaki $M^{2}$, Christoforidis $C^{1}$, Kazamias $G^{3}$, Pantouvaki $A^{4}$ \\ ${ }^{1}$ Department of Orthopaedic, General Hospital of Heraklion -Venizeleio, Crete, Greece \\ ${ }^{2}$ Department of Medical Oncology, General Hospital of Heraklion -Venizeleio, Crete, Greece \\ ${ }^{3}$ Department of Pathology, General Hospital of Heraklion - Venizeleio, Crete, Greece \\ ${ }^{4}$ Department of Physiotherapy, General Hospital of Heraklion- Venizeleio, Crete, Greece
}

*Corresponding Author: Grigorios Kastanis, kastanisg@ gmail.com

Received 15 September 2020;

Accepted 28 September 2020;

Published 04 October 2020

\begin{abstract}
Schwannomas or neurilemmomas are the most common benign tumors of the neural sheaths of the peripheral nerves. Incidence of these tumors in the hand accounts to $0.8-2 \%$. The majority of them appear as an isolated grown mass along the route of the nerves. Peripheral nerves benign tumors present many difficulties concerning the clinical diagnosis and only histopathological evidence provides the final diagnosis. In this case study, we present a 45 year old man with a grown asymptomatic mass on the volar surface of right hand (hypothenar) remained for two years and impinging the skin of the hand only the last months. Initial MRI results diagnosed the mass as gaglion cyst.

Histopathological examination revealed a schwannoma. We present this case as a rare manifestation of a schwannoma in hypothenar area of the hand. A literature review of diagnoses and therapeutic management is also presented.
\end{abstract}

Keywords: Schwannoma, neurilemmoma, hand, nerve sheath tumors.

\section{Introduction}

Schwannomas or neurilemmomas by definition are tumors of peripheral nerve sheaths, consisting of benign neoplastic Schwann cell origin. They are the most common type of benign tumor of peripheral nerves and their incidence varies between $0,8-5 \%$ of all soft tissue tumors in hand and wrist while the majority of them appear on the volar surface ${ }^{[1,2]}$. A schwannoma grows slowly, is initially asymptomatic for a long period of time while the symptoms begin with the increasing size of the tumor ${ }^{[3]}$. It is typically arised from a single fascicle of the nerve and at the time of growing, more fascicles are affected.

The majority of Schwannomas present as a solitary manifestation along the course of the nerve but multiple lesions with an incidence of $1 \%$ to $23 \%$ have been reported and might be presented as a part of Neurofibromatosis 2(NF2), Schwannomatosis and Carney complex ${ }^{[2,4]}$. Between genders the incidence of tumor is contiguous and is met mostly in 3rd and 6th decades of life ${ }^{[5]}$

Peripheral nerves benign tumors present many difficulties among the clinical diagnosis and only histopathological evidence provides the final diagnosis. In hand, they are usually confused with gaglion cyst or carpal tunnel syndrome ${ }^{[6]}$.

The aim of this study is to present a case with a growing mass on the volar surface of the hand (hypothenar), with impinging of the skin, which MRI examination revealed as a gaglion cyst initially, while the histopathological examination confirmed a schwannoma and to expound the literature among diagnoses and therapeutic management.

\section{Case Report}

A 45 years old male manual worker was presented to our day clinic from another department for an isolate lump at the volar surface of the right hand (dominant hand) and with a diagnosis of gaglion cyst. The lump was at the hypothenar area of the right hand and the patient referred pain and numbness at the ulnar side of ring digit during grasping object with his palm. (Fig 1) The mass started to grow two years earlier, was pain free and in smaller size, while the numbness started two months before. There was no family history of neurofibromatosis. We distinguished a mobile soft palpable mass over the hypothenar region in zone three at the palm. The Tinel's test over the mass was positive. The patient underwent magnetic reasonable imaging, which revealed a gaglion cyst at the hypothenar region of right hand. (Fig 2(a),2(b),2(c)) We suggested to the patient, total excision of the tumor for histopathological examination. Under regional anesthesia a longitudinal approach, approximately $6 \mathrm{~cm}$ centered upon the tumor mass, was performed. (Fig 3) The tumor was situated over hypothenar muscles (opponens digiti minimi and flexor digiti minimi brevis) and firmly attached to the superficial branch of ulnar nerve with a size of 25x16x10mm. (Fig4(a),4(b)) Histopathological examination of the 
tumor revealed a schwannoma. (Fig 5) Postoperatively, patient followed a rehabilitation program including passive and assistive movements of the little and ring finger initially, adding a progressive scheme of active exercises of fingers later. Neural tension exercises were also given concerning the location of tumor and possible complications. Three weeks after surgery, all symptoms were disappeared and patient returned to previous daily activities.

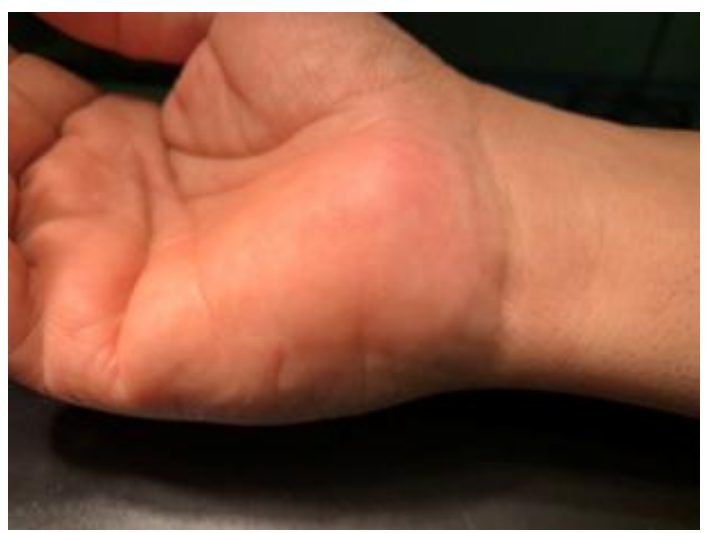

Figure 1: Palpable isolated mass on hypothenar region of the right hand (black arrow)

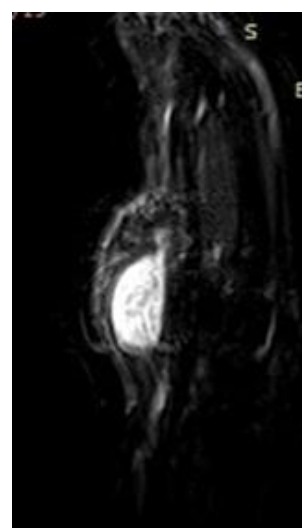

(a)

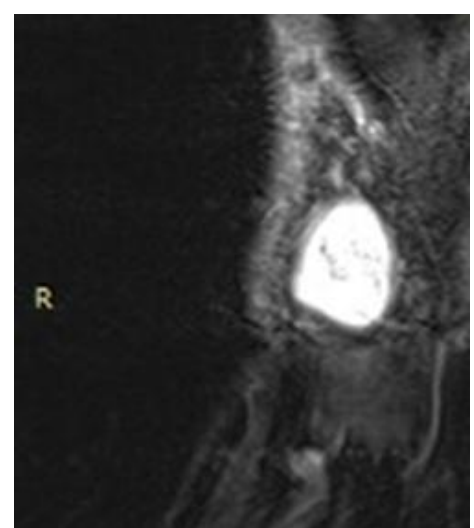

(b)

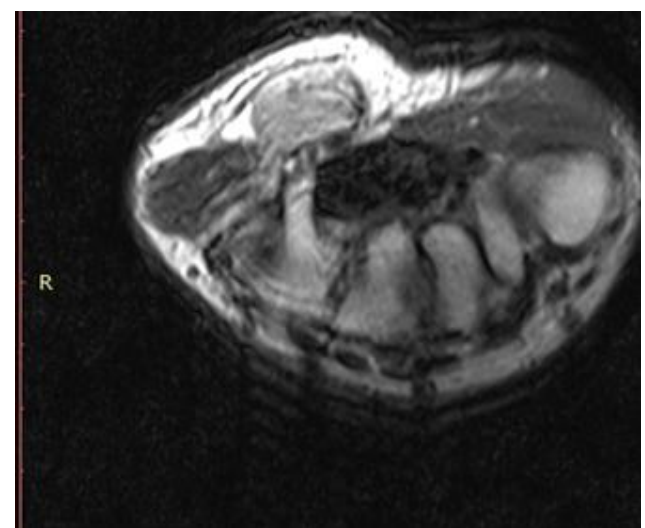

(c)

Figure 2: Preoperative MRI of the right hand (white arrow)

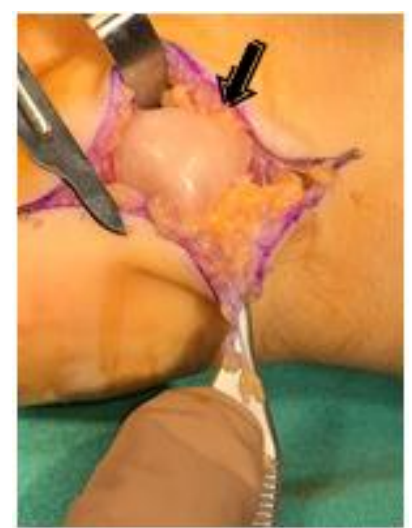

Figure 3: Intraoperative image of the tumor(black arrow)

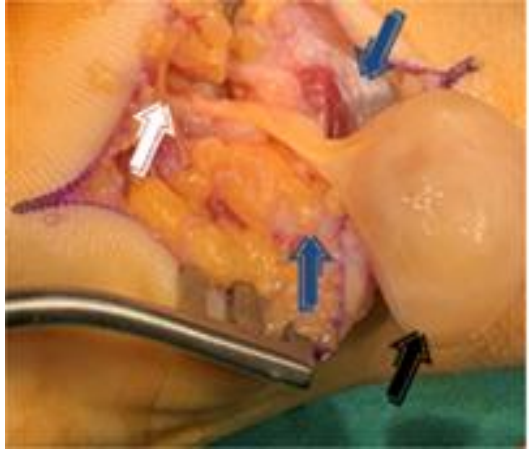

(a)

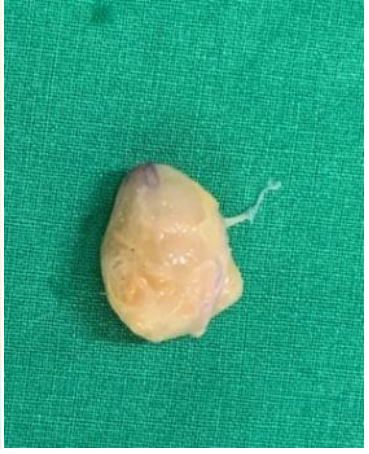

(b)

Figure 4: (a) Tumor (black arrow) situated over the hypothenar muscles (blue arrows) and attached to the superficial branch of ulnar nerve (white arrow), (b) Size of excited tumor $25 \times 16 \times 10 \mathrm{~mm}$ 


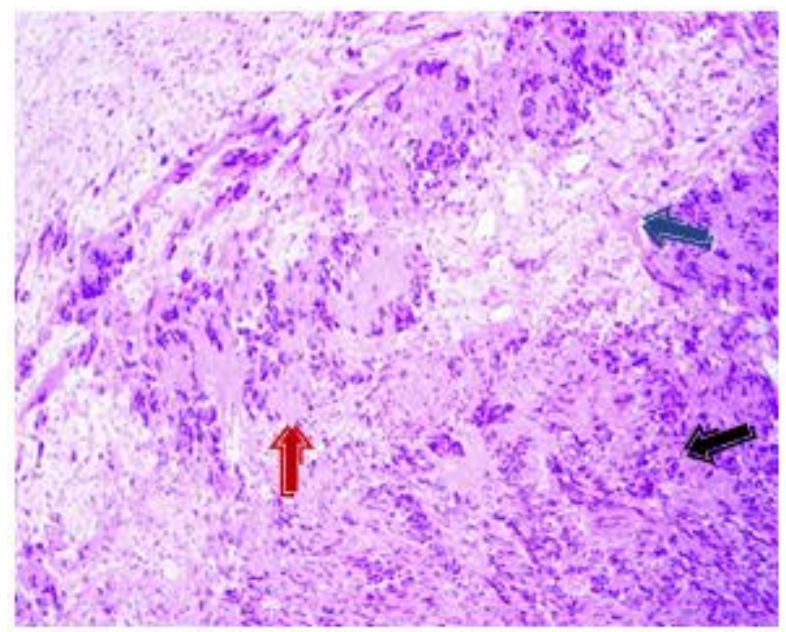

Figure 5: Histology of schwannoma with its characteristics pattern: Antoni A area (black arrow), Antoni B area (blue arrow) and Verocay body (red arrow).

\section{Discussion}

Schwannomas or neurilemomas are rare benign tumors but are defined as the most common peripheral tumors of the hand and wrist. Phalen GS reported 12 cases of Schwannoma localizated in hand and wrist, while Kehoe et al studying 104 cases, found only 30 cases localizated in hand and wrist ${ }^{[1]}$. The most affected peripheral nerves are the median and ulnar. Wrist and palm involvement is extremely rare and only a few cases have been reported in international literature with the most affected nerve to be median ${ }^{[2,5,15,21]}$.

Schwannomas are generally solitary, slowly growing mass and the period from the initial symptomatology until surgery varied from months to years ( 3 months to 37 years), while the size of the tumor can reach up to $4 \mathrm{~cm}$ before symptoms start from nerve compression ${ }^{[7]}$. These benign tumor became to be symptomatic from mechanical compression and presented with pain, swelling or sensory deficit. Ozdemir et al suggested that the onset of symptoms is associated with the location of the tumor rather than the size and patients with tumors grown in digital region of hand, proceed to medical examination earlier than those whose tumor is localized in the wrist and palm area ${ }^{[1]}$. In our case, the interval between appearance of the mass and the onset of symptomatology was two years and the surgery performed in two months after initial symptoms.

Etiology of solitary schwannomas is unclear because the majority of them are discovered promptly. It has been reported, in isolated cases of schwannoma, in association with foreign bodies, and there is a theory that micro trauma to the skin probably stimulates the growth of Schwann cells ${ }^{[11,12]}$. Our patient is manual worker(carpenter) and the continuous pressure from different instruments in the hypothenar region(micro trauma) might be the factor for the development of the tumor mass.

Due to the fact that these benign tumors appear as growing painless mass, the correct diagnosis presents many difficulties and in majority of cases can be misdiagnosed as another tumor ${ }^{[20]}$. Differential diagnosis should include: ganglion cyst (a theory related to degeneration of mucoid connective tissue had been proposed), neurofibroma, lipomas, xanthomas, giant cell tumors, granulomas and malignant tumors(squamous cell carcinoma) ${ }^{[10,11]}$. Kehoe et al, in 88 cases showed that preoperative diagnosis of schwannoma was correct only in one case ${ }^{[8]}$. Hems et al diagnosed preoperatively 7 cases in 10 patients, while similar results (5 patients to the 14 cases) were reported by Ozdemir ${ }^{[1,9]}$. In our case, it was initially diagnosed with clinical and magnetic reasonable imaging as a gaglion cyst.

However the correct clinical diagnoses of the tumor is difficult, magnetic reasonable imaging(MRI) and high-resolution sonography(HRS) can confirm information to support the diagnoses but are not $100 \%$ accurate to differentiating from other tumors in a large percentage of patients. MRI can provide useful information about location, morphological elements of tumor, defining origin, and appear the neurovascular elements ${ }^{[13]}$. Hems et al supported that MRI is unable to specifically differentiate the schwannomas from the other tumors (neurofibroma) and it is used to confirm the preoperative diagnoses and to help the surgeon to plan the surgical intervention ${ }^{[9]}$. On MRI, schwannomas appeared as middle low intensity signals at TI images and high intensity signals at $\mathrm{T} 2{ }^{[2]}$. On the contrary, high-resolution sonography is an important diagnostic tool offering information about the size, site basic features of the mass during static and dynamic positions. In HRS, the schwannoma is presented as a discrete, homogenous ovoid and hypoechoic mass, with a healthy nerve at the proximal and distal aspects of the mass ${ }^{[14,15]}$.

Surgical excision of the mass is the gold standard of the treatment. Bhattacharyya et al suggest excision of only symptomatic tumors or in cases in which during the follow-up the mass is growing ${ }^{[16]}$. The most important issue is to avoid damaging the nerve fibers during the epineural and endoneural dissection, and the most common postsurgical complication is paresthesia ${ }^{[19]}$. Recurrence of schwannoma is extremely rare, and has been appeared in different areas of the involved nerve of the same extremity but not in operated sites ${ }^{[18]}$. Ozdemir et al support that the recurrence may be as a result to incomplete excision of the tumor or misdiagnosis of multiple tumors as solitary ones. In our case, all the symptoms were disappeared in three weeks postoperatively without any complication and at the last examination in six months' time there was no clinical evidence of recurrence.

\section{Conclusion}

Schwannomas or neurilemomas are the most common benign peripheral neural tumors of the hand and wrist. Among the etiology of the tumor, the micro trauma might constitute a sequence. Meticulous medical history and clinical examination are crucial to come up a correct diagnosis. Imaging examinations (MRI, HRS) will provide useful information even though they don't have great accuracy for preoperative strategy management. Surgical treatment 
impacts in two factors, firstly the correct diagnosis and secondly, the relief of symptoms, therefore it remains the gold standard of treatment.

\section{Ethical Approval}

Our institution does not require ethical approval for reporting individual cases or case series.

\section{Consent Form}

Verbal informed consent was obtained from the patient for their anonymized information to be published in this article.

\section{Conflict of interest}

The authors declare that have no conflict of interest.

\section{Funding Statement}

There was no financial support for research and publication of this article.

\section{References}

[1] Ozdemir O, Ozsoy MH, Kurt C, et al. Schwannomas of the hand and wrist: long-term results and review of the literature. J Orthop Surg (Hong Kong). 2005; 13:267-72.

[2] Kütahya H, Güleç A, Güzel Y, Kacira B, Toker S. Schwannoma of the median nerve at the wrist and palmar region of the hand: a rare case report. Case Rep Orthop. 2013; 2013:950106. Doi: 10.1155/2013/950106.

[3] Adani R, Baccarani A, Guidi E, et al. Schwannomas of the upper extremity: diagnosis and treatment. Chir Organi Mov. 2008;92:85-8.

[4] Hasham S, Matteucci P, Stanley PR. Schwannomaרtosis: multiple schwannomas of the upper limb. J Hand Surg Br 2006;31:182-4 .

[5] Padasali PS, Shankaregowda VS, Kshirsagar SD. Median nerve schwannoma: a case and review of the literature. Asian J. of Neurosurgery 2015;10(3):212-215, doi: 10.4103/1793-5482.161178

[6] Aslam N, Kerr G. Multiple schwannomas of the median nerve: A case report and literature review. Hand Surg 2003;8:249-52

[7] Kang HJ, Shin SJ, Kang ES. Schwannomas of the upper extremity. J Hand Surg Br 2000;25:604-7.
[8] Kehoe NJ, Reid RP, Semple JC. Solitary benign peripheral-nerve tumours. Review of 32 years' experience. J Bone Joint Surg Br 1995;77:497-500.

[9] Hems TE, Burge PD, Wilson DJ. The role of magnetic resonance imaging in the management of peripheral nerve tumours. J Hand Surg Br 1997;22:57-60.

[10] Sandberg K, Nilsson J, Søe Nielsen N, Dahlin LB. Tumours of peripheral nerves in the upper extremity: A 22-year epidemiological study. Scand J Plast Reconstr Surg Hand Surg 2009;43:43-9.

[11] Lin A,Richands SW. Benign palmar schwannoma- a rare case in a handball player. BMJ Case Reports,2012; 2012: bcr0120125532 doi: 10.1136/bcr.01.2012.5532

[12] Kneitz H, W eyandt G, Meissner C, e t al. Dermal schwannoma (neurilemmoma): a peculiar foreign body reaction? Am J Dermatopathol 2010; 32: 367- 9.

[13] Aydin MD, Kotan D, Keles M. Acute median nerve palsy due to hemorrhaged Schwannoma: case report," Journal of Brachial Plexus and Peripheral Nerve Injury, 2007;2(19): 32-35.

[14] Stuart RM, Koh ES, Breidahl WH. Sonography of peripheral nerve pathology. AJR. 2004;182:123-9.

[15] Zardi EM,Vadala G, Buzzulini F, Laghi A, Afeltra A, Denaro V, Papapietro N. Imaging and surgical approach for a schwannoma of the hand. J Med Ultrasonics 2014;41(2):229-32, doi:10.1007/s10396-013-0495-7.

[16] Bhattacharyya AK, Perrin R, Guha A. Peripheral nerve tumors: management strategies and molecular insights," JournaL of Neuro-Oncology, 2004;69(1):335-349.

[17] Lee SH, Jung HG, Park YC, Kim HS, "Results of neurilemona treatment: a review of 78 cases," Orthopedics, 2001;24(10):977-980.

[18] Artico M, Cervoni L, Wierzbicki V, D'Andrea V, Nucci F. Benign neural sheath tumours of major nerves: characteristics in 119 surgical cases. Acta Neurochir (Wien) 1997; 139:1108-16.'

[19] Takase K, Yamamoto K, Imakiire A. Clinical pathology and therapeutic results of neurilemmoma in the upper extremity. J Orthop Surg (Hong Kong) 2004;12:222-5.

[20] Kececi Y, Gurler T, Gundogan H, Bilkay U, Cagdas A. Benign giant schwannoma located in the upper arm. Ann Plast Surg 1997; 39:100-2.

[21] Turcan S, Coskum E, Tuzun HY, Arsenishvili A, Can N, Cicek AF, Kulachi Y, Kurklu M. Schwannoma on the palmar surface of the hand: a rare case report. Hand Microsurg 2015;4(3):71.74 